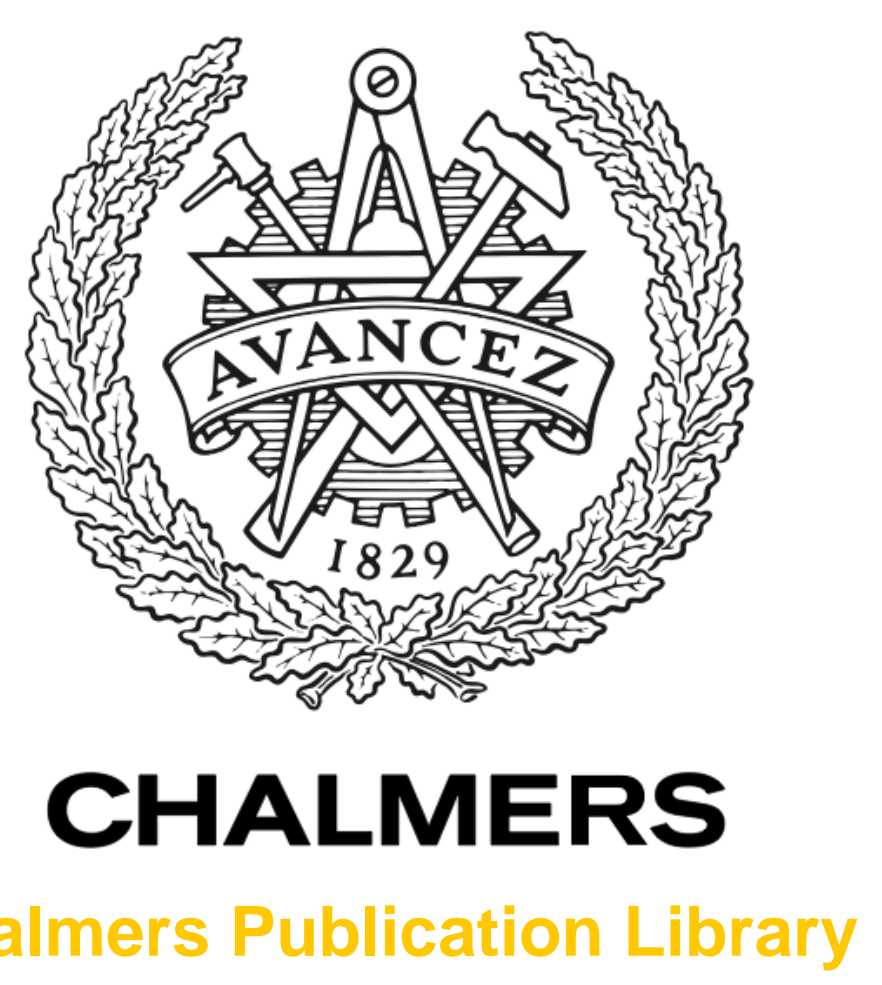

Chalmers Publication Library

Geometry-Based Stochastic Modeling and Estimation of Vehicle to Vehicle Channels

This document has been downloaded from Chalmers Publication Library (CPL). It is the author's version of a work that was accepted for publication in:

2014 IEEE International Conference on Acoustics, Speech, and Signal Processing, ICASSP 2014; Florence; Italy; 4 May 2014 through 9 May 2014 (ISSN: 15206149)

Citation for the published paper:

Beygi, S. ; Ström, E. ; Mitra, U. (2014) "Geometry-Based Stochastic Modeling and Estimation of Vehicle to Vehicle Channels". 2014 IEEE International Conference on Acoustics, Speech, and Signal Processing, ICASSP 2014; Florence; Italy; 4 May 2014 through 9 May 2014 pp. 4289-4293.

http://dx.doi.org/10.1109/ICASSP.2014.6854411

Downloaded from: http://publications.lib.chalmers.se/publication/195379

Notice: Changes introduced as a result of publishing processes such as copy-editing and formatting may not be reflected in this document. For a definitive version of this work, please refer to the published source. Please note that access to the published version might require a subscription. 


\title{
GEOMETRY-BASED STOCHASTIC MODELING AND ESTIMATION OF VEHICLE TO VEHICLE CHANNELS
}

\author{
Sajjad Beygi, Erik G. Ström*, Urbashi Mitra \\ School of Electrical Engineering, University of Southern California \\ *Dept. of Signals and Systems, Chalmers University of Technology \\ Emails: \{beygihar, ubli\}@usc.edu, erik.strom@chalmers.se
}

\begin{abstract}
In this paper, a geometry-based stochastic channel model (GSCM) for vehicle-to-vehicle (V2V) wireless communication is developed. The channel model reveals that the channel representation in delay-Doppler domain can be divided into four regions. In each region, the $\mathrm{V} 2 \mathrm{~V}$ channel can be modeled using a hybrid sparse/diffuse (HSD) model. Prior art on hybrid channel estimation for linear time-invariant channels is extended to the time-varying case. Furthermore, the effects of pulse shape leakage are explicitly determined and compensated. Simulation results shows that exploiting the $\mathrm{V} 2 \mathrm{~V}$ channel properties in the delay-Doppler domain, yields significantly improved channel estimates over unstructured approaches (more than $10 \mathrm{~dB}$ gain in SNR).
\end{abstract}

\section{INTRODUCTION}

Vehicle-to-vehicle (V2V) communications are central to future intelligent transportation systems (ITS), which will enable efficient and more safe transportation [1]. High fidelity wireless communications in high-speed and on-road environments is desired. Clearly, this imposes a challenge in designing robust vehicle-to-vehicle (V2V) communication systems to combat impairments incurred by rapidly changing wireless channels. There are several distinguishing features for the V2V channel in comparison to the cellular channel. For instance, antenna heights of both transmitter and receiver are low [2]. Furthermore, since both transmitter and receiver are moving, the $\mathrm{V} 2 \mathrm{~V}$ channel can be statistically stationary for a shorter time interval relative to cellular channel. Also, due to the high dependency of $\mathrm{V} 2 \mathrm{~V}$ channel on the geometry of the road, such as street intersections, tunnels [1,3], and the local physical environment such as urban, suburban, and rural areas [1,3], researchers have modeled different classes of $\mathrm{V} 2 \mathrm{~V}$ channels for these different cases.

In this work, we adopt and analyze the geometric stochastic channel model (GSCM) that was proposed from measurements in [4]. The contributions of our work are: $(i)$ we show that the channel representation in the delay-Doppler domain can be divided to four distinct regions and in each region, the channel can be modeled by a region-specific hybrid sparse/diffuse channel model; (ii) we show that the leakage effect in the delay-Doppler domain can be modeled as two independent leakage functions in the delay and Doppler directions, and the shape of leakage function is independent of the delay and Doppler value; (iii) we

This research was funded in part by one or all of these grants: ONR N00014-09-1-0700, NSF CNS-0832186, NSF CCF-1117896, CCF-0917343, CNS-1213128, AFOSR FA9550-12-1-0215, DOT CA-26-7084-00, Barbro Osher Pro Suecia Foundation, Ericsson's Research Foundation FOSTIFT-13:038, and Adlerbert Research Foundation. adapt the hybrid sparse/diffuse (HSD) channel estimator [8-11] to a time-varying/2D V2V channel model; and ( $i v)$ we robustify the channel estimator by explicitly compensating for pulse shape leakage at the receiver.

The rest of the paper is organized as follows: in Section 2, we present geometry-based V2V channel modeling. In Section 3, the signal model, channel representation in delay-Doppler, and leakage effect are presented. Then, in Section 4, the estimator for the HSD time-varying/2D channel model is presented. Section 5, we provide simulation results and we compare the performance of the estimators. Finally, Section 6 concludes the paper.

\section{GEOMETRY-BASED V2V CHANNEL MODELING}

In geometry-based stochastic channel modeling (GSCM) [4], an ensemble of point scatterers is placed according to a statistical distribution, assigned different channel properties, and used to determine the composite contribution at the receiver. Motivated by [1, 3, 4], we consider the following contributions to a V2V channel: $(i)$ the effective line-of-sight (LOS) component, which may contain the ground reflections, $(i i)$ discrete components generated from reflections of mobile scatterers (MD), e.g., other vehicles, $($ iii $)$ discrete components reflected from static scatterers (SD) such as bridges, large traffic signs, etc., and ( $i v)$ diffuse components (DI). Thus, the V2V channel impulse response can be written as

$$
\begin{aligned}
h(t, \tau)=h_{L O S}(t, \tau) & +\sum_{i=1}^{N_{M D}} h_{M D, i}(t, \tau) \\
& +\sum_{i=1}^{N_{S D}} h_{S D, i}(t, \tau)+\sum_{i=1}^{N_{D I}} h_{D I, i}(t, \tau),
\end{aligned}
$$

where $N_{M D}$ denotes the number of mobile discrete scatterers, $N_{S D}$ is the number of static scatterers and $N_{D I}$ is the number of diffuse scatterers. In the above representation, the multipath components can be modeled as $h_{i}(t, \tau)=a_{i} \delta\left(\tau-\tau_{i}\right) e^{-j 2 \pi \nu_{i} t}$, where $a_{i}$ is the complex channel gain, $\tau_{i}$ is the delay, and $\nu_{i}$ is the Doppler shift associated with path $i$ and $\delta(t)$ is the Dirac delta function.. The spatial distribution of the scatterer and the statistical properties of the complex channel gains are specified in [4] for a rural and highway environments. In particular, the complex channel gains are modeled as independent, complex Gaussian. The details about the spatial evolution of the gains are found in [4], but are omitted here since they are not important for this paper. Hereafter, we assume that movement is only along the $x$ axis and speeds are considered positive in the $x$-axis direction. Note that the only difference between a static scatterer (SD) and diffuse scatterer (DI) is the statistics of the reflected signal from the scatterer [4]. We will place an ensemble of each of these 


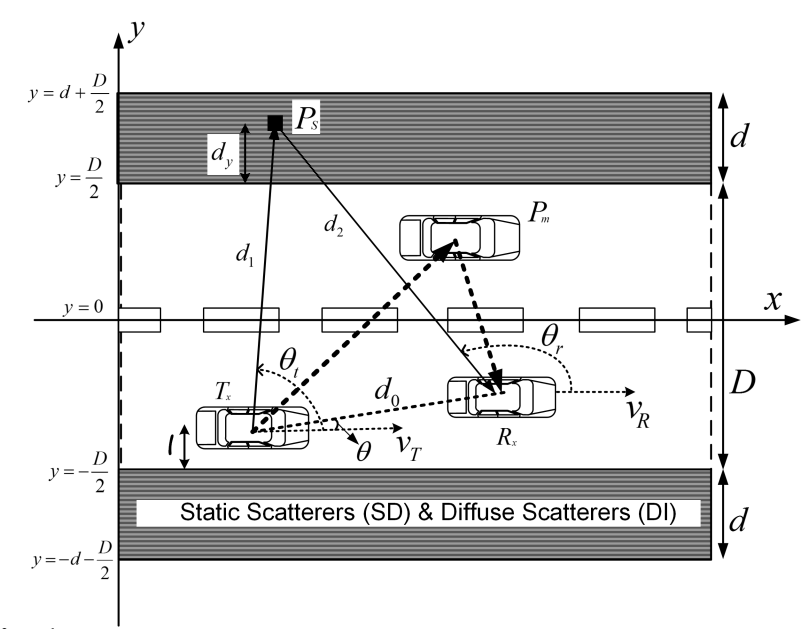

Fig. 1: Geometric representation for the transmission between two mobile vehicles in a road. Shaded area are diffuse components.

point scatterers in the geometry in Fig.1, and determine its delay and Doppler contributions. The overall Doppler shift for the path from the transmitter $T$ via the scatterer $P$ to the receiver $R$ can be written as [5]

$$
\nu\left(\theta_{t}, \theta_{r}\right)=\frac{1}{\lambda}\left[\left(v_{T}-v_{P}\right) \cos \theta_{t}+\left(v_{R}-v_{P}\right) \cos \theta_{r}\right],
$$

where $\lambda$ is the wavelength, $v_{T}, v_{P}$, and $v_{R}$ are the speed of the transmitter, scatterer, and receiver, respectively, and $\theta_{t}$ and $\theta_{r}$ is the angle of departure and arrival, respectively. The path delay is

$$
\tau=\frac{d_{1}+d_{2}}{c_{0}}
$$

where $c_{0}$ is the propagation speed, $d_{1}$ is distance from $T$ to $P$, and $d_{2}$ is the distance from $P$ to $R$. The path parameters $\theta_{t}$, $\theta_{r}, d_{1}$, and $d_{2}$ can easily be computed from the positions of $T, P$, and $R$. The delay-Doppler information of each component $(i)-(i v)$ can be specified by Equations (2) and (3) for the single-bounce of a general point scatterer. The most significant component of the V2V channel is the LOS, which will appear at $\tau_{0}=\frac{d_{0}}{c_{0}}$ and $\nu_{0}=\frac{1}{\lambda}\left(v_{T}-v_{R}\right) \cos (\theta)$ in the delay-Doppler domain representation of V2V channel (LOS Region 1 in Fig. 2). The scatterers that make up the diffuse component are assumed to be static $\left(v_{P}=0\right)$ and uniformly distributed in two strips of width $d$ parallel to the direction of travel [4] (Fig. 1). We observe from Fig. 2 that the contribution of the diffuse scatterers on the parallel strips are confined to a U-shaped area. For a different value of $y$, there will be a different $\mathrm{U}$-shaped curve in the delay-Doppler domain. To determine the geometry of Region 2, LOS Tail, we need to know $\Delta \tau$, the delay for LOS path $\tau_{0}$, and $v_{T}$ and $v_{R}$ the speed of transmitter vehicle and receiver vehicle, respectively. From the delay difference between the geometry of traveling path in two extreme cases, namely the LOS and diffuse scatterers from $d_{y}=d$ (see Fig.1), we can show that $\Delta \tau \approx \frac{2(d+D)-(2-\cos (\theta)) d_{0}}{c_{0}}$, where $0 \leq \theta \leq \frac{\pi}{2}$. Assuming that $v_{T}$ and $v_{R}$ are non-negative, the Doppler from the DI and SD scatterers in the shaded region in Fig. 1 are bounded by $\nu_{\max }=\frac{v_{T}+v_{R}}{\lambda}$ and $\nu_{\min }=-\frac{v_{T}+v_{R}}{\lambda}$. For their delay contribution, we have $\tau_{0}<\tau<\tau_{\max }$ where $\tau_{\max }-\tau_{0}$ is the maximum significant delay for the V2V channel. From Equation (2), we see that the components caused by a strong reflection from an MD scatterer (i.e., a vehicle) in the oncoming lane $\left(v_{P}<0\right)$ are

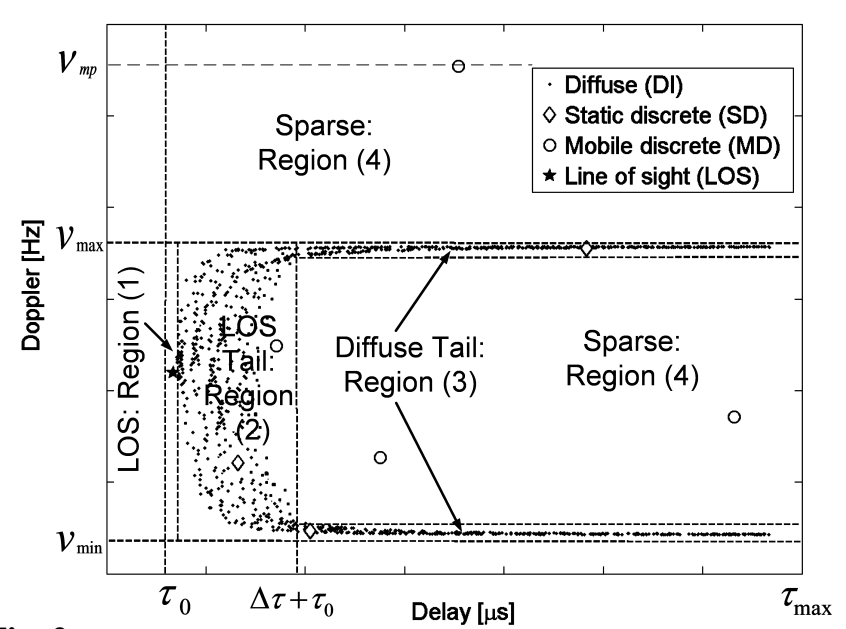

Fig. 2: Delay-Doppler domain representation of V2V channel. DelayDoppler spreading function is confined in a U-shape area.

located outside the Doppler region between $\nu_{\min }$ and $\nu_{\max }$. For example in Fig. 2, the Doppler shift $\nu_{m p}$ is due to a vehicle in the oncoming lane (opposite direction). SD scatterers are not moving $\left(v_{P}=0\right)$, and the corresponding Doppler will bounded by $\nu_{\min }$ and $\nu_{\max }$. Hence, SD scatters can be present in Regions 2 and 3 and Region 4. Based on the analysis for delay and Doppler values of each type of scatterers in this section, we conclude that we can divide the delay-Doppler representation of the channel into four key regions. The associated scatterers for each region are labeled in Fig. 2.

\section{SYSTEM MODEL}

We assume that there is single-user communication between two vehicles, as in Fig.1. The transmitted signal $x(t)$ is generated by the modulation of the transmitted pilot sequence $x[n]$ onto the interpolation filter $p_{t}(t)$ as, $x(t)=\sum_{n=-\infty}^{+\infty} x[n] p_{t}\left(t-n T_{s}\right)$, where $T_{s}$ is the sampling period. Note that this signal model is quite general and covers, e.g., OFDM signals. The signal $x(t)$ is transmitted over a $\mathrm{V} 2 \mathrm{~V}$ channel as described in the previous section. The received signal can be written as, $r(t)=\int_{-\infty}^{+\infty} h(t, \tau) x(t-\tau) d \tau+z(t)$. Here, $h(t, \tau)$ is the channel's time-varying impulse response, as defined in (1) and $z(t)$ is complex Gaussian noise. At the receiver, $r(t)$ is converted into discrete-time signal using an anti-aliasing filter $p_{r}(t)$ by, $r[n]=\int_{-\infty}^{+\infty} r(t) p_{r}\left(n T_{s}-t\right) d t$. An equivalent discrete-time channel $h_{l}[n, m]$ is described by the following relationship between the discrete-time signals $x[n]$ and $r[n]$ : $r[n]=\sum_{m=-\infty}^{+\infty} h_{l}[n, m] x[n-m]+z[n]$. Here $h_{l}[n, m]$ is the discrete time-delay representation of the observed channel. The relationship between the discrete-time-equivalent channel $h_{l}[n, m]$ and the continuous-time channel $h(t, \tau)$ is

$$
h_{l}[n, m]=\int_{-\infty}^{+\infty} \int_{\infty} h\left(t+n T_{s}, \tau\right) p_{t}\left(t-\tau+m T_{s}\right) p_{r}(-t) d t d \tau .
$$

We assume that $h_{l}[n, m]$ is causal with maximum delay $M-1$, i.e., $h_{l}[n, m]=0$ for $m \geq M$ and $m<0$. Then if we transmit $N_{r}+M-1$ samples of $x[n]$, we have,

$$
r[n]=\sum_{k=0}^{K-1} \sum_{m=0}^{M-1} H_{l}[k, m] x[n-m] e^{j \frac{2 \pi}{K} n k}+z[n],
$$


where $n=0,1, \ldots, N_{r}-1, K \geq N_{r}$, and

$$
H_{l}[k, m]=\frac{1}{K} \sum_{n=0}^{N_{r}-1} h[n, m] e^{-j \frac{2 \pi}{K} n k}, k=0,1, \ldots, K-1
$$

is the discrete-delay-Doppler spreading function [6].

Using Equation (4), the discrete-time impulse response for a single scatter (path) $h_{i}(t, \tau)=a_{i} \delta\left(\tau-\tau_{i}\right) e^{j 2 \pi \nu_{i} t}$ can be written as $h_{l, i}[n, m]=a_{i} e^{j 2 \pi \nu_{i} n T_{s}} \phi_{\tau}\left(m-\frac{\tau_{i}}{T_{s}}\right)$, where $\phi_{\tau}(m) \approx$ $\left.p_{t}(t) * p_{r}(t)\right|_{t=T_{s} m}$. This approximation is good if $\nu_{i}$ is not too large and if $p_{t}(t) * p_{r}(t)$ decays sufficiently fast, which is typically true for pulse shapes for $\mathrm{V} 2 \mathrm{~V}$ short range communication. Using the above definition of the discrete-delay-Doppler spreading function, we have

$$
\begin{aligned}
H_{l, i}[k, m] & =a_{i} \phi_{\tau}\left(m-\frac{\tau_{i}}{T_{s}}\right)\left(\frac{1}{K} \sum_{n=0}^{N_{r}-1} e^{j 2 \pi \nu_{i} n T_{s}} e^{-j \frac{2 \pi}{K} n k}\right) \\
& =a_{i} g\left(k-k_{i}, m-m_{i}\right)
\end{aligned}
$$

where $g(k, m)=\phi_{\nu}(k) \phi_{\tau}(m)$, with

$$
\phi_{\nu}(k)=\frac{\sin \left(\frac{\pi k N_{r}}{K}\right) \exp \left\{-j \pi\left(N_{r}-1\right) k / K\right\}}{K \sin \left(\frac{\pi k}{K}\right)},
$$

$k_{i}=\nu_{i} K T_{s}$, and $m_{i}=\frac{\tau_{i}}{T_{s}}$. Here, we assume that $T_{s}$ and $K$ is chosen such that $k_{i}$ and $m_{i}$ become integer numbers. We see that the leakage effect in the Doppler direction $\phi_{\nu}($.$) and in$ the delay direction $\phi_{\tau}($.$) act independently. Furthermore, the$ leakage in the Doppler direction is mainly due to the finite pilot sequence length $N_{r}$, and the leakage in the delay direction is due to the finite transmit bandwidth $\left(\approx 1 / T_{s}\right)$. One can show, using a Taylor series expansion of the two functions $\phi_{\nu}\left(k-k_{i}\right)$ and $\phi_{\tau}\left(m-m_{i}\right)$, that when the filters $p_{t}($.$) and p_{r}($.$) are ideal$ lowpass filter (sinc-filters), $\phi_{\nu}\left(k-k_{i}\right)$ and $\phi_{\tau}\left(m-m_{i}\right)$ both decay linearly (polynomially of order 1 ) with respect to distance from $k_{i}$ and $m_{i}$, but when these filters are the family of rootraised-cosine filters, $\phi_{\tau}\left(m-m_{i}\right)$ decays polynomially of order 3 with respect to distance from $m_{i}$. Therefore, the leakage in the Doppler direction is more severe than that in the delay direction. Due to the linearity of the Fourier transform, we can conclude that

$$
H_{l}[k, m]=\sum_{i} a_{i} g\left(k-k_{i}, m-m_{i}\right)
$$

where the summation is over the LOS component and all the $N_{M D}+N_{S D}+N_{D I}$ scatters.

Let us define $\mathbf{c}_{l} \in \mathbb{C}^{(M K \times 1)}$ and $\mathbf{c} \in \mathbb{C}^{(M K \times 1)}$ as follows $\mathbf{c}_{l}=\left[H_{l}[0,0], H_{l}[1,0], \ldots, H_{l}[K-1, M-1]\right]^{T}$ and $\mathbf{c}=\operatorname{vec}\{\mathbf{C}\}$, where $[\mathbf{C}]_{k, m}=a_{i} \delta\left[k-k_{i}\right] \delta\left[m-m_{i}\right]$, and $\delta[k]$ is the Kronecker delta function. Then, we can rewrite Equation (7) in matrix form as $\mathbf{c}_{l}=\mathbf{G c}$. Here $\mathbf{G} \in \mathbb{C}^{(M K \times M K)}$ is a matrix with elements $[\mathbf{G}]_{i, j}=g\left(r_{2}-r_{1}, q_{2}-q_{1}\right)$, where $i=q_{1} K+r_{1}$ and $j=q_{2} K+r_{2}$ with $0 \leq r_{1} \leq K-1$ and $0 \leq r_{2} \leq K-1$.

\subsection{Receiver Processing}

The source vehicle transmits a sequence of $N_{r}+M-1$ pilots, $x[n]$, over the channel. If we collect the $N_{r}$ received samples in a column vector $\mathbf{r}=\left[r[0], r[1], \ldots, r\left[N_{r}-1\right]\right]^{T}$, we have the following matrix representation:

$$
\mathbf{r}=\mathbf{X} \mathbf{c}_{l}+\mathbf{z}=\mathbf{X G c}+\mathbf{z}
$$

The noise vector is $\mathbf{z} \sim \mathcal{C N}\left(\mathbf{0}, \sigma_{z}^{2} \mathbf{I}_{N_{r} \times N_{r}}\right)$, and the data matrix $\mathbf{X}$ is an $N_{r} \times M K$ block matrix of the form $\mathbf{X}=$ $\left[\mathbf{X}_{0}, \ldots, \mathbf{X}_{M-1}\right]$. Here, each block $\mathbf{X}_{m} \in \mathbb{C}^{\left(N_{r} \times K\right)}$ is of the form $\mathbf{X}_{m}=\mathbf{D}_{m} \boldsymbol{\Omega}$, where $\mathbf{D}_{m} \in \mathbb{C}^{\left(N_{r} \times N_{r}\right)}$ is a diagonal matrix given by $\mathbf{D}_{m}=\operatorname{diag}\left\{x[-m], \ldots, x\left[N_{r}-m-1\right]\right\}$ for $(0 \leq m \leq M-1)$, and $\Omega \in \mathbb{C}^{\left(N_{r} \times K\right)}$ is a Vandermonde matrix, $\boldsymbol{\Omega}_{i, j}=\omega_{j-1}^{i-1}$, with $\omega_{k}=e^{j \frac{2 \pi k}{K}}$ for $0 \leq k \leq K-1$. We assume the use of a well-behaved pilot sequence such that $\mathbf{X}^{\mathbf{H}} \mathbf{X} \succ \mathbf{0}$ ( $\mathbf{X}$ is positive definite), thus, the LS estimate of $\mathbf{c}_{L S}=\mathbf{G}^{-1}\left(\mathbf{X}^{\mathbf{H}} \mathbf{X}\right)^{-1} \mathbf{X}^{\mathbf{H}} \mathbf{r}$ is a sufficient statistic [7] for the channel. Therefore, without loss of generality, for the purposes of channel estimation, we consider the observation model to be $\mathbf{c}_{L S}=\mathbf{c}+\sqrt{\boldsymbol{\Sigma}}^{-1} \mathbf{n}$, where $\boldsymbol{\Sigma}=\frac{1}{\sigma_{z}^{2}} \mathbf{G}^{H} \mathbf{X}^{H} \mathbf{X G} \succ 0$ is defined as a SNR matrix and $\mathbf{n}$ is normalized complex Gaussian noise. Moreover, we assume that the pilot sequence is such that $\Sigma$ is diagonal then the noise vector $\sqrt{\Sigma}^{-1} \mathbf{n}$ in the LS estimate has independent entries. This assumption greatly simplifies the channel estimation problem. In fact, when the channel has independent entries, an element-wise estimation approach, rather than a joint one, is optimal. The loss incurred due to assuming orthogonal training when the sequence is not white is characterized in [9] and can be described by an SNR loss.

\subsection{Hybrid Sparse/Diffuse Model for V2V Channel}

Consider the regions specified in Fig.2, the delay-Doppler spreading function for every region in general can be modeled by hybrid sparse/diffuse (HSD) combination as

$$
\mathbf{c}=\mathbf{c}_{s}+\mathbf{c}_{d}
$$

where $\mathbf{c}_{s}$ is the sparse part including the LOS and discrete components such MD or SD, and $\mathbf{c}_{d}$ represents the diffuse part. The model for the distribution of $\mathbf{c}_{s}$ is given by $\mathbf{c}_{s}=\mathbf{a}_{s} \odot \mathbf{b}_{s}$ where $\odot$ is the Schur product, $\mathbf{a}_{s}$ represents the amplitude (energy) of discrete components and is treated as an unknown deterministic vector, and $\mathbf{b}_{s}$ is a vector whose elements are 0 or 1 , which are drawn i.i.d from Bernoulli model $\mathcal{B}(p)$ where $p$ is the probability of a discrete scatterer. The diffuse vector, $\mathbf{c}_{d}$, is distributed as $\mathbf{c}_{d} \sim \mathcal{C} \mathcal{N}\left(0, \boldsymbol{\Sigma}_{d}\right)$, where $\boldsymbol{\Sigma}_{d}$ is diagonal. The size of the vectors depends on the sizes of each regions as shown in Fig. 2.

Region 1 - LOS: In this region, only the direct path propagation and ground reflection play an important role. LOS components can show up in any Doppler value in the Region 1. Thus $\mathbf{c}_{s}$ represents the LOS and $\mathbf{c}_{d} \approx \mathbf{0}$.

Region 2 - LOS Tail: The energy of the coefficients decays exponentially in the delay direction and we know that the amplitudes of the coefficients in the Doppler direction are statistically independent [3, 4]. We treat $\mathbf{c}_{s}$ as a deterministic unknown vector. The diffuse vector is distributed as $\mathbf{c}_{d} \sim \mathcal{C N}\left(0, \boldsymbol{\Sigma}_{d}\right)$, where $\boldsymbol{\Sigma}_{d}=\boldsymbol{\Sigma}_{0} \otimes \mathbf{I}$ is diagonal. Here $\boldsymbol{\Sigma}_{0}$ is diagonal and is the correlation matrix associated with each row in the delay direction in Region 2, and $\otimes$ denotes the Kronecker product. The size of the identity matrix is specified by the number of discretization points (or number of rows) in the Doppler direction, $K$.

Region 3 - Diffuse Tail: As depicted in Fig. 2, the Doppler spread of the components in this region is very small compared to the Doppler resolution, thus we assume a common Doppler value for components in this region. The model can be developed in a similar approach as we have done for Region 2.

Region 4 - Sparse: Components are only due to the discrete scatters. Therefore, $\mathbf{c}_{d}=\mathbf{0}$. 


\section{CHANNEL ESTIMATION}

4.1. Review Of Hybrid Sparse/Diffuse Channel Estimator In [8. 9], we have developed estimation strategies for the static HSD model. In particular, we have proposed the estimator, for the scenario where the covariance matrix of the diffuse part $\mathbf{c}_{d}, \boldsymbol{\Sigma}_{d}$ is known at the receiver, whereas the vector of sparse coefficients $\mathbf{c}_{s}$ and the sparsity level $p$ are treated as unknown deterministic parameters. In general, the value of diffuse part $\mathbf{c}_{d}$ given the estimation of sparse part $\hat{\mathbf{c}}_{s}$, can be estimated as $\hat{\mathbf{c}}_{d}=\boldsymbol{\Sigma}_{d}\left(\boldsymbol{\Sigma}_{d}+\boldsymbol{\Sigma}^{-1}\right)^{-1}\left(\mathbf{c}_{L S}-\hat{\mathbf{c}}_{s}\right)$. Here $\boldsymbol{\Sigma}$ is the SNR matrix, defined in Section 3.1. Then, the generalized threshold estimator (gThresh Estimator) [8] of the delay-Doppler spreading vector, given the LS estimation of delay-Doppler spreading vector, is given as $\hat{\mathbf{c}}_{s}=\mathbf{c}_{L S} \odot \hat{\mathbf{b}}_{s}$, where $\left[\hat{\mathbf{b}}_{s}\right]_{k}=\mathbb{1}\left(\left|\left[\mathbf{c}_{L S}\right]_{k}\right|^{2} \leq \alpha\left(\left([\boldsymbol{\Sigma}]_{k, k}\right)^{-1}+\left[\boldsymbol{\Sigma}_{d}\right]_{k, k}\right)\right)$, where $\mathbb{1}($.$) is indicator function and \alpha=\frac{1-p}{p}$.

\subsection{Proposed Channel Estimation Algorithm}

Our proposed channel estimation algorithm can briefly stated as:

Step 1: (LS Estimation and Leakage Compensation) Collect $N_{r}$ samples of received signal $r[n],\left(n=0, \ldots, N_{r}-1\right)$ in a vector, then given $N_{r}+M-1$ available pilots, compute the least-square solution $\mathbf{c}_{L S}$. We compensate for the leakage effect by applying the LS estimator weighted by the matrix $\mathbf{G}$ in Equation (8).

Step 2: (Region Specification) Assuming knowledge of street geometry, $d_{0}, \theta$, and speeds of both transmitter $v_{T}$ and receiver $v_{R}$, specify Regions 1 to 4 as described in Fig. 2 of the computed LS solution, then vectorize the channel within each region. Note that to vectorize each region, pick rows from the matrix form of each region and stack them in a vector. In practice, the regions must be estimated from the data, and this is planned to be addressed in future work.

Step 3: (Refinement) Apply the gThresh Estimator from Section 4.1 to refine the LS solution.

The signal model derivation in Section 3 provides the needed tools to extend the estimators in [8] to the time-varying case, that is, $H_{l}[k, m]$ captures both delay and Doppler information. In vectorizing $H_{l}$ to create the effective channel vector $\mathbf{c}_{l}$, we can use the methods of [8] (summarized in Section 4.1). We observe that the structure of $\boldsymbol{\Sigma}_{d}$ is a direct consequence of the modeling of the time-varying channel and its effects on the description of the diffuse part.

\section{NUMERICAL SIMULATION}

In this section, we simulate the mean squared error for $\mathrm{V} 2 \mathrm{~V}$ channel estimation employing our new proposed methods. To simulate the channel, we consider a geometry with $x_{\min }=$ $-200 \mathrm{~m}$ and $x_{\max }=200 \mathrm{~m}$, road width $D=18 \mathrm{~m}$, and the length of the diffuse strip around the road $d=10 \mathrm{~m}$. The locations of the transmitter and receiver are chosen randomly in this geometry with distance $d_{0}=20 \mathrm{~m}$ from each other. The speed of transmit and receive vehicles are assumed as $v_{T}=100$ $(\mathrm{km} / \mathrm{h})$ and $v_{R}=105(\mathrm{~km} / \mathrm{h})$, respectively. It is assumed that the number of MD scatterers $N_{M D}=5$ (driving in the same direction with transmit and receive vehicles), SD scatterers $N_{S D}=10$ and DI scatterers $N_{D I}=1000$. Furthermore, we have considered $f_{c}=5.9 \mathrm{GHz}, T_{s}=50 \mathrm{~ns}, N_{r}=32768$, and $M=512$. The statistical parameter values for different scatterers are selected to be those reported in Table I in [4], which

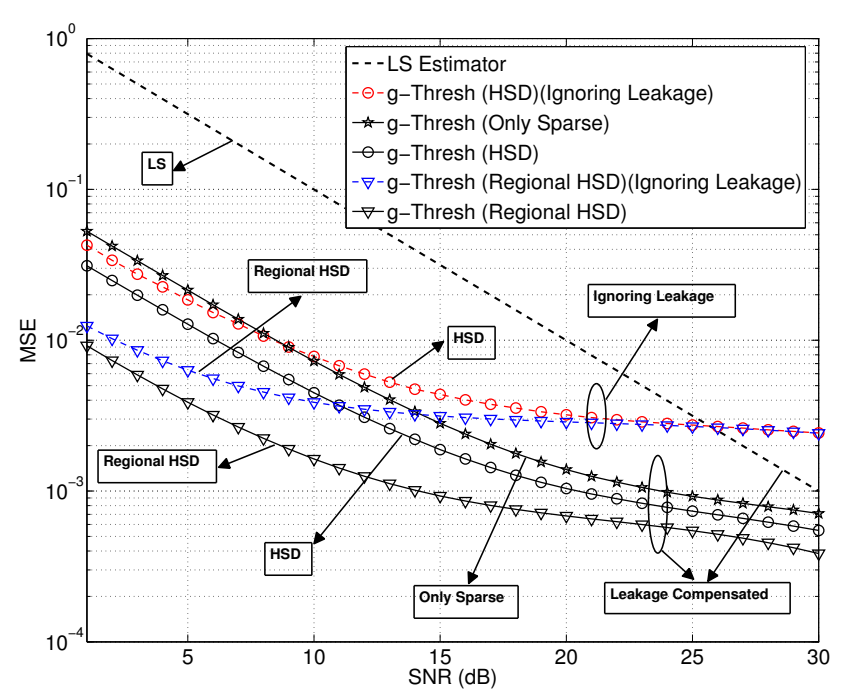

Fig. 3: MSE of the channel estimators. The expression "Ignoring Leakage" in the legend means that leakage effect is not compensated, i.e., $\mathbf{G}=\mathbf{I}$ is assumed in the channel estimation algorithm.

are computed by practical experiment and measurement data analysis. In Fig. 3 depicts the result for Monte Carlo simulation for estimation of channel using g-Threshold estimator and LS estimator. We have considered three different scenarios with reasonable channel representation in the delay-Doppler domain (Note that the real channel is not changed between the scenarios, but only the algorithms assumptions about the channel is changed). In the first scenario, the entire channel is assumed to follow the sparse model (Only Sparse), in the second scenario (HSD model), it is assumed that the entire channel follows the common HSD model, and finally in the third scenario (Regional HSD), we have applied the proposed algorithm by applying HSD model for the four regions defined in the Section 3.2. The MSE is defined as $E\left\{\|\hat{\mathbf{c}}-\mathbf{c}\|_{2}^{2}\right\}$, where $\hat{\mathbf{c}}$ is the estimated channel vector. We added complex white Gaussian noise $\mathbf{z}$ whose variance was adjusted to achieve a prescribed receive signal-tonoise ratio (SNR) defined as SNR $=\frac{E\left\{\|\mathbf{r}-\mathbf{z}\|_{2}^{2}\right\}}{E\left\{\|\mathbf{z}\|_{2}^{2}\right\}}$ It is clear that there is at least 5 to $15 \mathrm{~dB}$ performance enhancement when we consider the structural information of the channel in different regions relative to other prior information. Furthermore, there are two curves that represent the leakage effect for HSD and Regional HSD modeling. In these two cases, we have ignored leakage compensation, namely assumed $\mathbf{G}=\mathbf{I}$. We can observe that leakage effect reduces the performance severely, about 5 to $10 \mathrm{~dB}$ in low SNR.

\section{CONCLUSIONS}

In this paper, we developed a the $\mathrm{V} 2 \mathrm{~V}$ channel representation in delay-Doppler domain using geometry-based stochastic channel modeling. We showed that channel can be summarized into four regions and in each region channel can be modeled as HSD channel modeling. We characterized the leakage and showed it can be decomposed as a leakage in the Doppler direction and a leakage in delay direction independently. Next, we extended the HSD channel estimator of [8] to the time-varying/2D V2V channel model and we robustified the channel estimator by explicitly compensating for pulse shape leakage at the receiver. The simulation results showed that the proposed algorithm enhanced the performance of the LS estimator more than $10 \mathrm{~dB}$ for low to relatively high SNR. 


\section{REFERENCES}

[1] D. W. Matolak, "V2V communication channels: State of knowledge, new results, and whats next," in Commun. Tech. for Veh., pp. 1-21, Springer, 2013.

[2] A. Paier, J. Karedal, N. Czink, C. Dumard, T. Zemen, F. Tufvesson, A. F. Molisch, and C. F. Mecklenbrauker, "Characterization of vehicle-to-vehicle radio channels from measurements at $5.2 \mathrm{GHz}$," Wireless personal commun., vol. 50, no. 1, pp. 19-32, 2009.

[3] A. Molisch, F. Tufvesson, J. Karedal, and C. Mecklenbrauker, "A survey on vehicle-to-vehicle propagation channels," IEEE Trans. Wireless Commun., vol. 16, no. 6, pp. 12-22, 2009.

[4] J. Karedal, F. Tufvesson, N. Czink, A. Paier, C. Dumard, T. Zemen, C. F. Mecklenbrauker, and A. F. Molisch, "A geometry-based stochastic MIMO model for vehicle-tovehicle communications," IEEE Trans. Wireless Commun., vol. 8, no. 7, pp. 3646-3657, 2009.

[5] J. Karedal, N. Czink, A. Paier, F. Tufvesson, and A. Molisch, "Path loss modeling for vehicle-to-vehicle communications," IEEE Trans. Veh. Technol., vol. 60, no. 1, pp. 323-328, 2011.

[6] A. F. Molisch, "Wireless communications," Wiley press, 2nd ed., 2010.

[7] E. L. Lehmann and G. Casella, "Theory of point estimation," Springer, 2nd ed., 2005.

[8] N. Michelusi, U. Mitra, A. Molisch, and M. Zorzi, "UWB sparse/diffuse channels, part I: Channel models and bayesian estimators," IEEE Trans. Signal Process., vol. 60, no. 10 , pp. 5307-5319, 2012.

[9] N. Michelusi, U. Mitra, A. Molisch, and M. Zorzi, "UWB sparse/diffuse channels, part II: Estimator analysis and practical channels," IEEE Trans. Signal Process., vol. 60, no. 10 , pp. 5320-5333, 2012.

[10] P. Schniter, "A Message-Passing Receiver for BICMOFDM Over Unknown Clustered-Sparse Channels," IEEE J. Sel. Topics Signal Process., vol. 5, no. 8, pp. 1462-1474, 2011.

[11] C. Carbonelli, S. Vedantam, and U. Mitra, "Sparse channel estimation with zero tap detection," IEEE Trans. Wireless Commun., vol. 6, no. 5, pp. 1743-1763, May 2007. 